\title{
EDITORIAL
}

\section{Usefulness of Multimodal Investigations of the Choline Pathway}

\author{
Denis Rommel, Thierry P. Duprez \\ Department of Radiology, Université Catholique de Louvain, Louvain-la-Neuve, Belgium
}

$\mathrm{W}$ e feel very appropriate the present comments of Sandi Kwee and colleagues [1] on our experimental work comparing total choline concentration at H-MRS and choline uptake at $\left[{ }^{18} \mathrm{~F}\right]$-fluoromethylcholine $(\mathrm{FCH})[2]$. As stated by Kwee et al., the so-called total choline peak (tCho) adds different metabolites having a common quaternary ammonium chemical group, prominently choline (Cho), phosphocholine (PC), and glycerophosphocholine [3]. Concentration and contribution of those different molecules can only be determined by high-resolution spectra at very high field strength from cellular extract in experimental settings. We fully agree with Kwee and colleagues when they hypothesize that tissue concentration of PC should have putatively better correlation to FCH uptake than tCho did. However, an a priori governing our work was to perform investigational experiments in a pre-clinical setting, therefore using an in vivo model and a clinical $3 \mathrm{~T}$ imager to allow easier translation to human patients' cohorts in a further step. The major penalty we had then to assume using such system was its inability to separate the different sub-peaks of the 'full' tCho peak. However, we remain strongly convinced that the tCho peaks we obtained at $3 \mathrm{~T}$ significantly reflected tumor physiology as $\mathrm{PC}$ has been identified as the major choline component of ${ }^{1} \mathrm{H}$ spectra $[4,5]$. Thus far, the correlation between $\left[{ }^{18} \mathrm{~F}\right]$-fluoromethylcholine uptake with tCho peak should remain an accurate indicator of what the correlation between FCH uptake and specific PC peak would have been. Furthermore, Al Saffar et al. observed that decrease in tCho and decrease in PC levels measured by ${ }^{31} \mathrm{P}-\mathrm{MRS}$ were similar in MDAMB-231 cells (human breast carcinoma) treated by an anticancer drug and thereby concluded that the decrease in tCho peak was mainly due to the drop in PC, as major component of the tCho peak [6]. Ultimately, we share the opinion that further experimental/pre-clinical studies comparing different choline imaging techniques in a synergistic way are mandatory to fully elucidate the complexity of the Kennedy pathway.

\section{References}

1. Kwee S, Ernst T (2010) Letter to the editor Molecular Imaging and Biology

2. Rommel D, Bol A, Abarca-Quinones J et al. (2010) Rodent rhabdomyosarcoma: comparison between total choline concentration at H-MRS and [(18)F]-fluoromethylcholine uptake at PET using accurate methods for collecting data. Mol Imaging Biol. doi:10.1007/s11307-009-0283-3

3. Govindaraju V, Young K, Maudsley AA (2000) Proton NMR chemical shifts and coupling constants for brain metabolites. NMR Biomed 13:129-153

4. Gillies RJ, Morse DL (2005) In vivo magnetic resonance spectroscopy in cancer. Annu Rev Biomed Eng 7:287-326

5. Glunde K, Bhujwalla ZM (2007) Choline kinase alpha in cancer prognosis and treatment. Lancet Oncol 8:855-857

6. Al-Saffar NM, Troy H, Ramírez de Molina A et al (2006) Noninvasive magnetic resonance spectroscopic pharmacodynamic markers of the choline kinase inhibitor MN58b in human carcinoma models. Cancer Res $66: 427-434$ 